\title{
Non-Destructive Evaluation of an Infusion Process using Capacitive Sensing Technique
}

\author{
Yang Yang, Thomas Vervust, Frederick Bossuyt, Jan Vanfleteren \\ Center for Microsystems Technology, IMEC and Ghent University, Belgium \\ Gabriele Chiesura, Geert Luyckx, Joris Degrieck \\ Department of Materials Science and Engineering, Ghent University, Belgium
}

Markus Kaufmann

Sirris Leuven-Gent Composites Application Lab, Leuven, Belgium

\begin{abstract}
In this study, a capacitive sensing based non-destructive evaluation technique is applied to a vacuum assisted resin infusion process for the fabrication of glass fibre reinforced composites, as such different steps of the fabrication process (the injection of resin, the curing and the post curing) of a composite part can be better understood to increase the quality of the fabricated part and reduce the fabrication costs. An interdigital coplanar capacitive sensor was designed, fabricated, and embedded in the glass fibre reinforced composites. Experimental data clearly shows different stages of the resin infusion process: Flow front of the resin to capacitive sensor marked by rapid increase of capacitance; domination of ionic conduction at the early stage of the cure when the resin is still in a liquid state; the vitrification point, indicating a transition of the resin from a gelly state to a glassy state, marked by the relatively big decrease in capacitance; further polymerization during post-curing, marked by a peak in capacitance at the beginning of post-curing cycle, and finally the completion of the cure marked by the saturation of capacitance to a final value. The different phenomena observed during the experiment can be used as a tool for in situ on-line monitoring of composites cure.
\end{abstract}

\section{INTRODUCTION}

Fibre Reinforced Polymer(FRP) is becoming a valid alternative to many traditional heavy metal industries because of its high specific stiffness over the more classical construction metals. Among the various manufacturing techniques, Liquid composite moulding (LCM), and especially the Vacuum Assisted Resin Infusion process(VARI) is becoming a common tool for large composite part production. For instance, the traditional hand-layup process is getting replaced by VARI with the key motivation being health improvement of working environment of manufacturing plant due to lower volatile component emissions and less hazard contact between the operator and the materials. Besides, better control of the final component thickness is another advantage of VARI in comparison to hand layup process. Other benefit of VARI includes more economically advantageous than other process such as autoclave, since no expensive tooling is needed and cheaper raw materials in comparison to expensive pre-pregs [1].

With the increasing difficulty in composite manu- facturing process introduced by more complex composite shapes and manufacturing parameters (temperature, pressure, time and etc.), a method to optimize the manufacturing process is imposed such that different manufacturing steps (e.g. the injection of resin, the curing and post curing stage) can be understood to increase the quality of the produced part while reducing manufacturing costs. An important stage during manufacturing of a composite part is certainly the curing phase: during which the polymer constitute of composites cross-links, results in the solidification and hardening of whole composite structure. By controlling the curing phase one can obtain a superior quality component and, as a consequence, will increase the reliability and optimize the design of the part, leading to a decrease of the life cycle cost. Several methods have been proposed to characterize the cure kinetics and monitor the cure process, for instance Differential Scanning Calorimetry (DSC), Dynamic Mechanical Analysis (DMA), Ultrasonic Techniques, Raman Spectroscopy, and Dielectric Analysis (DEA)[2][3][4]. DEA is identified as an effective tool 
for on-line monitoring of composites cure, as the technique can be applied to different processing environment such as ovens, presses and autoclaves in a nondestructive manner[5]. Most of the other techniques are more suitable for a laboratory environment under ideal conditions.

In this work, a flexible interdigital capacitive sensor was designed, fabricated, and embedded in the glass fibre reinforced composites fabricated by a vacuum assisted resin infusion process. Experimental data clearly shows different stages of the resin infusion process: Flow front of the resin to capacitive sensor marked by rapid increase of capacitance; domination of ionic conduction at the early stage of the cure when the resin is still in a liquid state; the vitrification point, indicating a transition of the resin from a gelly state to a glassy state, marked by the relatively big decrease in capacitance; further polymerization during post-curing, marked by a peak in capacitance at the beginning of post-curing cycle, and finally the completion of the cure marked by the saturation of capacitance to a final value. The different phenomena observed during the experiment can be used as a tool for in situ on-line monitoring of composites cure.

\section{EXPERIMENTAL}

\subsection{Principle of capacitive sensing technique}

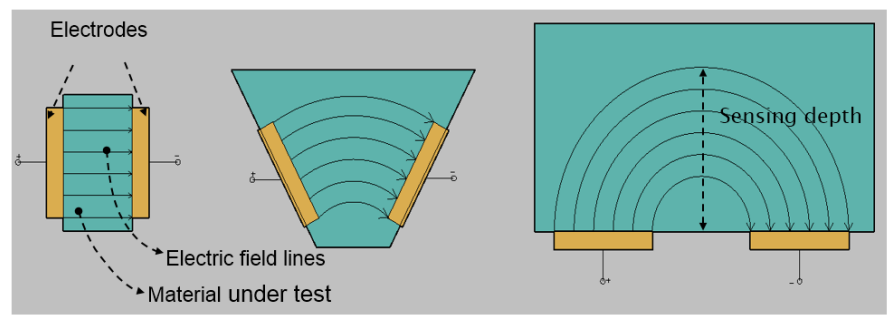

Figure 1: Transition from parallel plate capacitor (left) by opening up the face-to-face parallel electrodes(middle) until coplanar fringing field capacitor (right)

Capacitive sensing technique is often applied to study the dielectric properties of material, which is commonly referred to as dielectrometry or dielectric analysis [2]. This principle can be extended to cure degree monitoring of composite: during the curing of thermosetting polymer matrix composite, the mobility of dipoles (since most reactive molecules are dipolar) and free-moving ions (introduced during the synthesis of the resin) gets restricted, leading to decreased mobility of rotational dipoles and translational ions and are represented in the change of real relative permittivity and loss tangent. Monitoring of these changes allows us to identify different stages of the curing process. Materials dielectric property $\varepsilon^{*}$, is represented in a complex form:

$\varepsilon^{*}=\varepsilon^{\prime}-j \varepsilon^{\prime \prime}$

The real part $\varepsilon^{\prime}$ is referred to as real relative permittivity or dielectric constant: it expresses the capacity of a material to store the electrical energy. The imaginary part $\varepsilon^{\prime \prime}$ is referred to as the dielectric loss factor: it expresses the energy loss caused by the dielectric medium. They are dependent on polarization of the permanent dipole moment and translational ionic groups.

Most common capacitive sensor is based on the use of parallel plate electrodes or interdigital electrodes. For a parallel plate system, the material under test (MUT) is sandwiched between two electrodes to form a capacitor. Care should be taken when using parallel plate system: the distance between the electrodes should be kept constant throughout the experiment; A/d ratio should be reasonably large for the impedance measurement system to give enough sensitivity; the electrodes should be present on both sides of the mould (or other production environment). Interdigital electrode refers to a digit-like or finger-like periodic pattern of parallel in-plane electrodes used to build up the capacitance or impedance associated with the sensitive coating or embedding material, into which the electric field penetrates. Interdigital sensor, in contrast to parallel plate electrodes, has both anode and cathode on the same plane. Compare to the parallel plate system, the geometry of the system is more stable, as both electrodes are now in the same 2D plane. Moreover, due to the property of coplanar of electrodes, one side access to the material is advantageous, this makes embedding the sensor less troublesome in many cases. Fig. 1 shows the transition of parallel plate capacitor to a coplanary interdigital sensor and illustrates interdigital sensor's principle of operation.
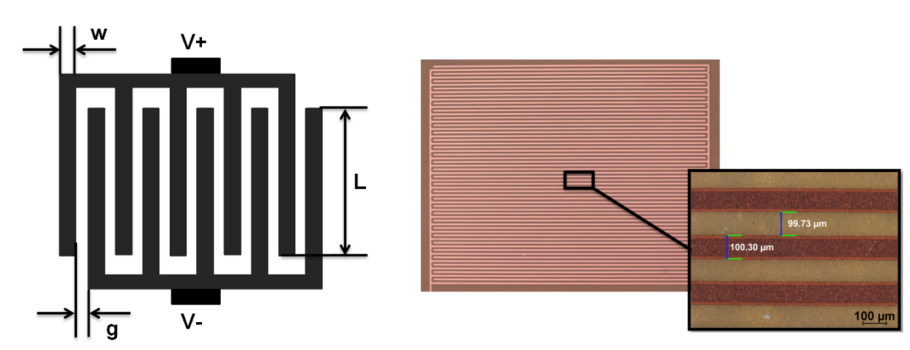

Figure 2: Simplified schematic of an interdigital capacitive sensor (left): where w stands for the width of each finger, $g$ stands for the gap (spacing) between fingers, and L stands for the finger length, and a home-made sample (right) realized by flexible circuit board technology

The simplified geometry of an IDC is shown in Fig. 2(left). When an electric potential is applied between the anode and cathode of the sensor, an electric field is created between the fingers, and extended to the space on both sides of the finger structure to a certain distance. The distance that the majority of the fringing electric field is able to reach is usually called the penetration depth of the sensor, determined by the spatial wavelength of the structure. Further details on interdigital sensor can be found from paper of Mamishev et al [6]. To establish a relationship between capacitance value and permittivity, these parameters 
were studied by mathematical models, Finite Element Analysis and experimental measurements. FEA and model proposed by Igreja et al [7] agree well with the experimental work; model proposed by H. Engan [8] has a large deviation from experimental result, as the model includes only one dielectric in the formula, whereas for the capacitive sensor at least two dielectric were present (pi substrate of the capacitive sensor and MUT).

The interdigital sensor, used in this study, is fabricated in-house by patterning copper structure on flexible substrate (PI/PET/PEN) via lithography and wet etching process, OSP (typical thickness $0.15-0.3$ micron) treatment is then applied on top of electrodes to protect the sensor surface from oxidation. Fig. 2(right) shows the fabricated capacitive sensor. The resulted sensor is $68 \mu \mathrm{m}$ thick, and $180 \mathrm{~mm}^{2}$ in size.

\subsection{Experimental setup}

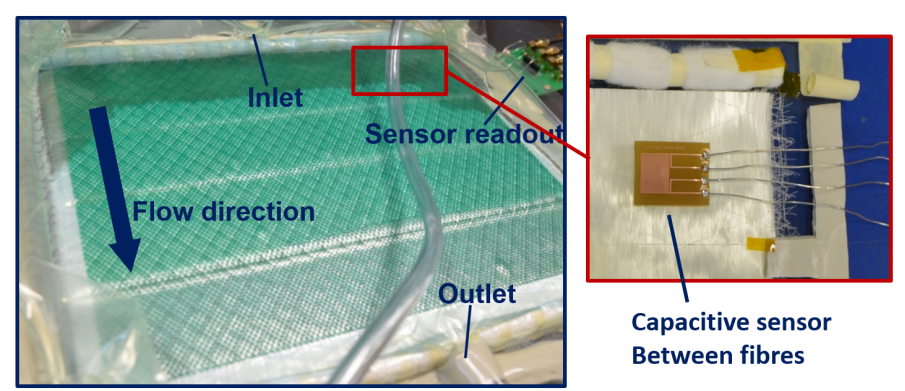

Figure 3: Experimental setup for the vacuum assisted resin infusion process, with the capacitive sensor embedded in between glass fibre reinforcement of the composites

A vacuum assisted resin infusion process to fabricate glass fibre reinforced composites is shown in Fig. 3. 6 layers of glass fibre textile were layered up on top of a flat glass mould, the plate was sealed on the 4 edges with vacuum bag. An interdigital sensor and a temperature sensor were embedded between the middle layers. The epoxy matrix system used in this study (from Momentive), is a two part system consisting of the EPIKOTE MGS RIMR 135 resin and the EPIKURE MGS RIMH 137 hardener. The mixing ratio is $100: 30$ by weight. $300 \mathrm{~g}$ of RIMR 135 (epoxy) and 90g of RIMH137 (hardener) were thoroughly mixed by a mechanical blender and degassed, and infused to the vacuum bagging system with a tube connected to the inlet of the system, the outlet was connected with a tube to vacuum, and once the resin reaches the outlet, both tubes on the inlet and outlet side was clamped to stop the resin flow. The glass fibre reinforced composite is cured at room temperature for $24 \mathrm{~h}$, followed by a post curing profile in the oven for $16 \mathrm{~h}$. A capacitive sensor, and two thermocouple were installed for capacitive and temperature measurement respectively throughout the curing. An HP 4284A Precision LCR meter performed the capacitive measurement with frequency sweep ranging from $100 \mathrm{~Hz}$ to $1 \mathrm{MHz}$ with 10 points per decade. The time required per sweep was approximately 16 seconds. One thermocouple was installed next to the capacitive sensor for local temperature measurement, and another one outside the vacuum bag for ambient temperature measurement.

\section{RESULTS AND DISCUSSIONS}

Capacitive data for the resin cured at $20^{\circ} \mathrm{C}$ are shown in Fig. 4. At the beginning of cure, we observe huge increase in capacitance value at around minute 35: indicating that the resin has reached the capacitive sensor, and flown completely over the capacitive sensor (marked by the maxima value). Zooming into the figure allow us to see that the capacitive value reaches maxima in 2 minutes, with the dimension of the capacitive sensor, we are able to calculate the resin flow velocity.

The huge increase in capacitance at the beginning is caused by electrode polarization [9]. As the resin starts off as liquid with low viscosity, the ionic impurities (introduced to the system during the synthesis of the resin) in the resin are able to move freely. Ion impurities accumulate at the electrode surfaces, causing interfacial polarization at the electrodes, leads to the large increase in capacitance dominating at lower frequencies. As the cure progresses, cross-linking between monomers or oligomers is continued, the polymer network is formed and expanded. The resin system becomes more viscous and the translational diffusion of ionic component gets restricted. Electrode polarization phenomena disappears first at higher frequencies, and gradually shifts to lower frequencies (Fig. 4). At around 12 hours into the cure, a relatively steep decrease in capacitance is observed. During the cure, as the resin becomes more viscous, the intermolecular forces that must be overcome to polarize become larger, the rotational diffusion of dipole moment is not able to follow the excitation signal at higher frequencies, and needs longer time for the reorientation and relaxation, marked by the steep decrease in real permittivity indicates the transition of the resin from a gelly state into a glassy state, and is referred to gelation point or vitrification point [10]. Worth mentioning during the $24 \mathrm{~h}$ curing stage, no obvious change of resin temperature is observed: resin temperature is following ambient temperature and no indication of degree of cure can be observed by temperature measurement.

Capacitive and temperature data for the post curing at 80 are shown in Fig. 5. During post-curing, the heating up of the oven leads to an increase in resin temperature. A huge increase in capacitance is observed in the beginning of post curing, after reaching the maxim, a rapid decrease of capacitance was seen, and the slope became smaller until decrease to almost zero. The overshoot of capacitance indicates further polymerization within the resin, when the temperature 


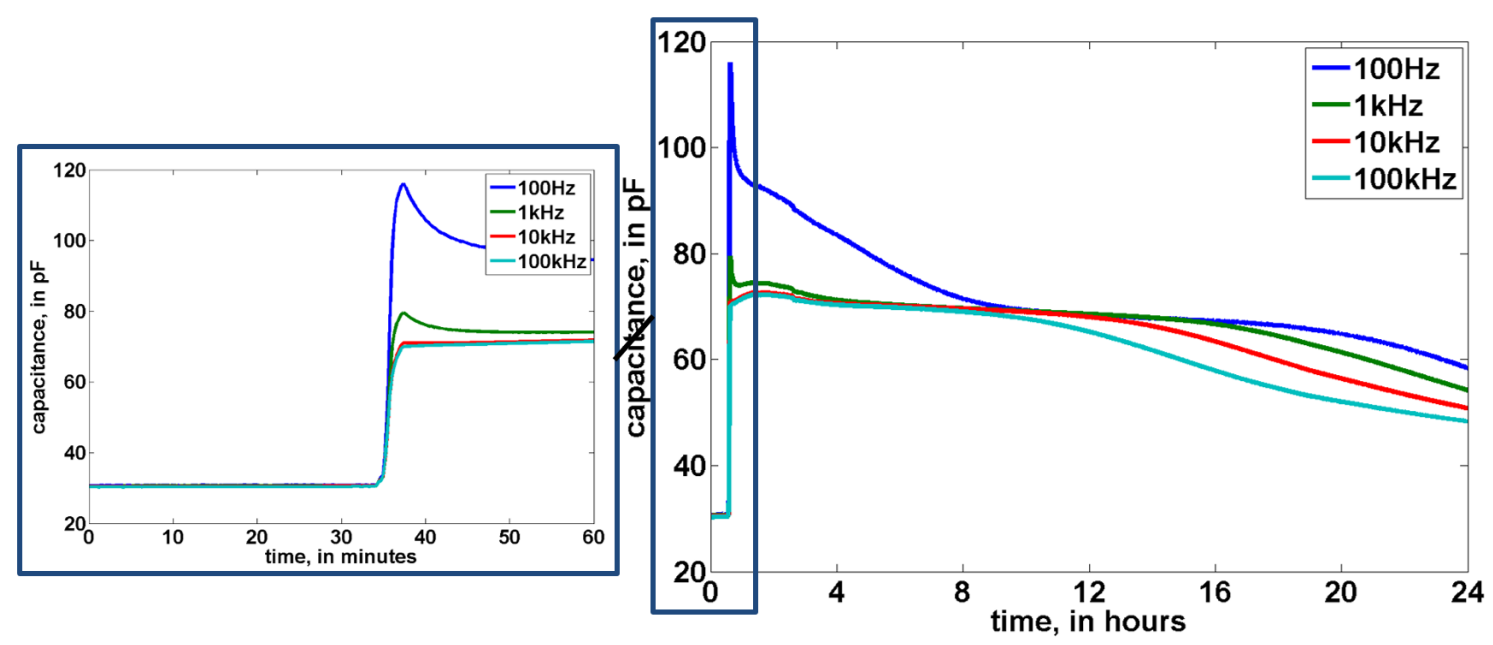

Figure 4: Capacitive measurement of beginning of curing(left) and whole curing phase(right)

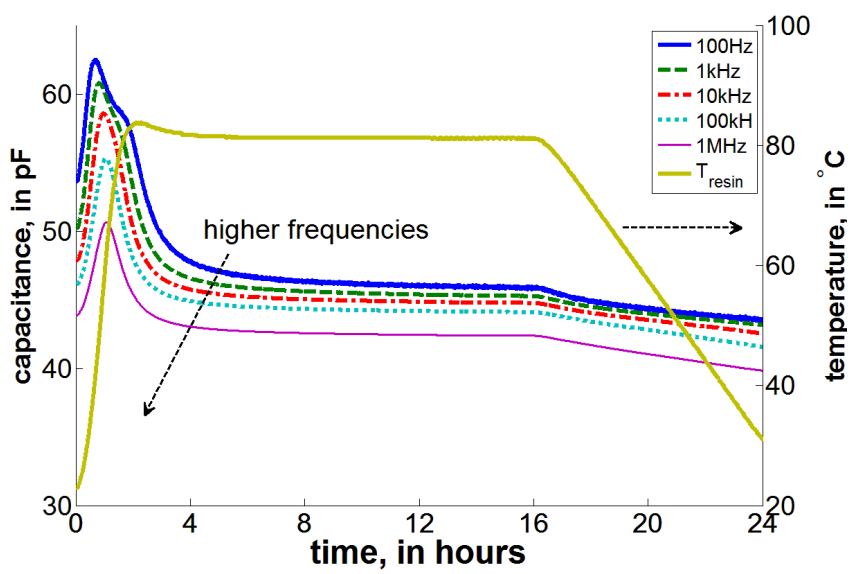

Figure 5: Capacitive and temperature measurement from post curing phase

is raised above glass transition temperature $(\mathrm{Tg})$. At the meantime, however, little confidence in temperature measurement could be obtained while temperature measurement follows the programmed heating profile of the oven. After the peak, capacitance values decrease and saturate to nearly stable values since the oven is kept at $80^{\circ} \mathrm{C}$. After $16 \mathrm{~h}$, the oven is shut down, and the decrease in temperature causes the drop in capacitance, as the dielectric property is temperature dependent, but the change in absolute value is considered insignificant compared to the peak observe at the beginning of post-curing or change during the curing stage. As can be seen from the plot, the capacitance measurement gives information about resin that is mostly related to degree of cure rather than the influence of the ambient environment, whereas the temperature measurement reveals little information about cure itself.

\section{CONCLUSIONS}

In this work, a flexible interdigital capacitive sensor was designed, fabricated, and embedded in the glass fibre reinforced composites. Experimental data clearly shows different stages of the resin infusion process: Flow front of the resin to capacitive sensor marked by rapid increase of capacitance; domination of ionic conduction at the early stage of the cure when the resin is still in a liquid state; the vitrification point, indicating a transition of the resin from a gelly state to a glassy state, marked by the relatively big decrease in capacitance; further polymerization during postcuring, marked by a peak in capacitance at the beginning of post-curing cycle, and finally the completion of the cure marked by the saturation of capacitance to a final value. The different phenomena observed during the experiment can be used as a tool for in situ on-line monitoring of composites cure.

Developed capacitive sensor is applicable not only to a laboratory scale for research purpose, but also to an industrial scale for real manufacturing monitoring purpose. The capacitive sensor can either be embedded inside the composite as an intermediate laminate layer, or be external to the composite; for instance, to be embedded on one side of the mould, vacuum bag plate or other types of surface with direct contact to the composites, or embedded as a thin film layer being part of the whole composite structure on the surface of the structure. In case of the sensor is embedded in the composite stack (either on the surface, or internally), the sensor is not only useful for the manufacturing monitoring of the composites, but also useful as a potential tool for the composites ageing monitoring during the service or maintenance phase. For instance, the ageing of polymer due to ultraviolet (UV) radiation will degrade the performance of the composites. The chemical composition of the polymer will be changed due to the ageing, this change will reflect in a change of dielectric property, which can be detected by the sensor. With the sensor embedded in the structure, costly manual inspection in a regular base in a harsh environment, for instance offshore wind turbines on the ocean, can be replaced by intelligent sensor based system that updates the central computer (or operator) in a timely base. The manufacturer is able to follow up the condition of their wind turbines $24 / 7$ and is able to diagnose a problem in an early phase prior to the damage. 


\section{ACKNOLWLEDGE}

The authors thank S. Dunphy, S. Van Put, and K. Dhaenens for the fabrication of capacitive sensors, J. Windels for his advice and assistance with hardware related issues. The research leading to these results has received funding from the Flemish Agency for Innovation by Science and Technology (IWT) through the program for Strategic Basic Research (SBO) under grant agreement n 120024 (Self Sensing Composites).

\section{REFERENCES}

[1] E. Poodts, G. Minak, E. Dolcini, L. Donati, $\{\mathrm{FE}\}$ analysis and production experience of a sandwich structure component manufactured by means of vacuum assisted resin infusion process, Composites Part B: Engineering 53 (0) (2013) $179-186$.

[2] J. D. Menczel, R. B. Prime, Thermal analysis of polymers : fundamentals and applications, John Wiley, Hoboken, N.J., 2009.

[3] J. Monni, P. Niemel, L. Alvila, T. T. Pakkanen, Online monitoring of synthesis and curing of phenolformaldehyde resol resins by raman spectroscopy, Polymer 49 (18) (2008) 3865-3874.

[4] F. Lionetto, F. Montagna, A. Maffezzoli, Ultrasonic transducers for cure monitoring: design, modelling and validation, Measurement Science and Technology 22 (12) (2011) 124002.

[5] G. M. Maistros, I. K. Partridge, Monitoring autoclave cure in commercial carbon fibre/epoxy composites, Composites Part B-Engineering 29 (3) (1998) 245-250.

[6] A. V. Mamishev, K. Sundara-Rajan, Y. Fumin, D. Yanqing, M. Zahn, Interdigital sensors and transducers, Proceedings of the IEEE 92 (5) (2004) 808-845.

[7] R. Igreja, C. J. Dias, Analytical evaluation of the interdigital electrodes capacitance for a multilayered structure, Sensors and Actuators A: Physical 112 (2-3) (2004) 291-301.

[8] H. Engan, Excitation of elastic surface waves by spatial harmonics of interdigital transducers, IEEE Transactions on Electron Devices 16 (12) (1969) 1014-1017.

[9] V. F. Lvovich, Impedance spectroscopy : applications to electrochemical and dielectric phenomena, Wiley, Hoboken, N.J., 2012.
[10] G. M. Maistros, C. B. Bucknall, Modeling the dielectric behavior of epoxy-resin blends during curing, Polymer Engineering and Science 34 (20) (1994) 1517-1528. 